\title{
A Bayesian statistical analysis of human T-cell lymphotropic virus evolutionary rates
}

\author{
Philippe Lemey ${ }^{\mathrm{a}, *}$, Oliver G. Pybus ${ }^{\mathrm{b}}$, Sonia Van Dooren ${ }^{\mathrm{a}}$, Anne-Mieke Vandamme \\ ${ }^{\mathrm{a}}$ Rega Institute for Medical Research, Minderbroedersstraat 10, Katholieke Universiteit Leuven, B-3000 Leuven, Belgium \\ ${ }^{\mathrm{b}}$ Department of Zoology, University of Oxford, South Parks Road, Oxford OX1 3PS, UK
}

Received 14 January 2004; accepted 29 April 2004

Available online 30 November 2004

\begin{abstract}
HTLV is a genetically-stable retrovirus that is considered to have evolved partly in concert with human migrations. Its rate of evolution is low and therefore, difficult to estimate reliably. In the first part of this study, we provide an improved estimate of HTLV evolutionary rate using anthropological calibration of phylogenetic nodes. We investigate two different anthropological calibrations using a Bayesian method that implements a relaxed molecular clock model and can combine data from multiple genes. The analysis shows that the two calibrations are compatible. In the second part, we develop a Bayesian statistical model to combine and compare the anthropology-based estimates of evolutionary rate with a rate recently calculated using pedigree data from vertically HTLV-infected families. We compare the statistical power of the two estimates and show that the current pedigree estimate, although resulting in considerably higher evolutionary rates, is too statistically weak to warrant a re-examination of the commonly used anthropology-based estimates. Statistical uncertainty burdens HTLV rate estimates based on both anthropological calibrations and on pedigree data; the former method rests on an untested assumption, whilst that latter is affected by small sample sizes.
\end{abstract}

(C) 2004 Elsevier B.V. All rights reserved.

Keywords: HTLV; Evolutionary rate; Bayesian modelling; Anthropology; Molecular clock; Dating; Co-divergence; Phylogeny

\section{Introduction}

The human T-cell leukemia/lymphotropic virus (HTLV) is a complex oncogenic retrovirus that has frequently crossed the species barrier between simians and humans (Liu et al., 1996; Salemi et al., 1998; Vandamme et al., 1994). Like the related human immunodeficiency virus (HIV), HTLV possesses an error-prone reverse transcriptase, providing it with the potential for high rates of evolution. However, HTLV does not appear to exploit this capacity since natural isolates show a remarkable genetic stability. This is evident when the within-host genetic diversity of HIV and HTLV is compared. In one study, no mutations were observed in proviral HTLV-1 isolated from the same person over several years (Gessain et al., 1992). In contrast, considerable genetic diversification can be observed during

\footnotetext{
* Corresponding author. Tel.: +32 16332160; fax: +32 16332131 .

E-mail address: philippe.lemey@uz.kuleuven.ac.be (P. Lemey).
}

the course of a single HIV infection (Holmes et al., 1992; Shankarappa et al., 1998). The low sequence diversity of HTLV is thought to arise from the replication dynamics of the virus. HTLV probably undergoes a limited number of replications during infection; high proviral loads are primarily maintained by clonal expansion of the infected cells (Wattel et al., 1995). Since mitosis uses cellular DNA polymerases, HTLV-infected daughter cells contain highfidelity copies of the parental provirus.

Virus populations are often sampled at different points in time. If a statistically significant number of genetic differences accumulate between time points then the sampled population is defined as a measurably evolving population (MEP) (Drummond et al., 2003). Many viruses, particularly RNA viruses, evolve very rapidly and therefore, satisfy this condition. A number of statistical methods have been developed to utilize the temporal information contained in MEPs (Drummond et al., 2002; Pybus and Rambaut, 2002; Rambaut, 2000); these methods are 
commonly used to investigate the origin of viral epidemics, to date epidemic and evolutionary events, and to infer past population dynamics (Korber et al., 2000; Lemey et al., 2003; Pybus et al., 2001, 2003). The MEP concept can also be applied to slowly-evolving organisms, provided that sequences are sampled at sufficiently different time points. For example, 'ancient DNA' has been reliably obtained from organic material preserved for tens of thousands of years (Drummond et al., 2003). Unfortunately, no reliable "fossil genomes" of substantial sequence length are available for slowly-evolving viruses, so methods developed for MEPs cannot be used. However, epidemic events can still be dated if it can be shown that the virus and its host share a common evolutionary history (McGeoch et al., 2000). Known dates in the evolutionary history of the host are applied to the equivalent event in the history of the virus, thereby providing a calibration for the evolutionary rate of the virus. This 'codivergence' method requires phylogenetic concordance between host and pathogen (Holmes, 2003).

Two types of genetic information are now available to investigate the tempo of HTLV evolution.

(i) Anthropology-based estimates: For the primate T-cell lymphotropic viruses (PTLV), there is at least partially a relationship between the phylogeny of the virus and the geographic location of its host (Vandamme et al., 1994). Specifically, one node in the HTLV phylogeny appears to coincide with the earliest migration of humans to Melanesia and Australia, and this node has been frequently used to estimate a timescale for PTLV evolution (Meertens and Gessain, 2003; Meertens et al., 2003; Salemi et al., 2000; Van Dooren et al., 2001; Yanagihara et al., 1995). To satisfy the molecular clock assumption, these studies usually discarded data inconsistent with this null hypothesis (Meertens and Gessain, 2003; Meertens et al., 2003; Salemi et al., 2000; Van Dooren et al., 2001). In addition, previous analyses have not incorporated the uncertainty in the anthropological dates in a statistical rigorous way. The extent to which HTLV genetic variation is representative of human migration patterns is largely unknown; hence such calibrations obviously depend heavily on the accuracy of the anthropological date being used.

(ii) Pedigree-based estimates: A recent pedigree-based study attempted to estimate the HTLV evolutionary rate without recourse to anthropological events (Van Dooren et al., 2004). Two different regions in the HTLV-1 genome, LTR and env, were sampled from infected individuals belonging to eight families. In total, only five substitutions were observed during 419 to 1109 years of evolution, confirming the genetic stability of HTLV. Maximum likelihood estimates of the evolutionary rate in this study appeared to be higher than those based on anthropological calibrations. Although the pedigree-based study does not satisfy the definition of a MEP, knowledge of birth dates, ages and sampling dates for the infected families introduces temporal information that is almost as useful as seriallysampled sequence data.
In the first part of this study, we provide improved anthropology-based estimates of HTLV rates using a Bayesian method that relaxes the rate-constancy assumption and takes into account the uncertainty in calibration dates. We also examine the consistency of two different anthropological calibrations. In the second part, we develop a Bayesian statistical model to compare and combine the anthropology-based phylogenetic results with the recent pedigree-based data.

\section{Materials and methods}

\subsection{Consistency of anthropological calibrations}

We investigated the consistency of two different anthropological calibrations using an alignment consisting of all PTLV coding regions (gag, protease, polymerase, envelope and tax). This data set contains 27 strains, including PTLV-1 and PTLV-2. We did not include PTLV3 strains because they have a significantly different nucleotide composition (tested using TREE-PUZZLE; Strimmer and von Haeseler, 1996). Maximum likelihood and Bayesian phylogenies were estimated using the GTR substitution model with gamma-distributed rate variation in PAUP4b10 and MrBayes, respectively (Huelsenbeck et al., 2000; Swofford, 1998). The topologies of the ML and Bayesian MAP trees were identical.

The molecular clock hypothesis was tested using the likelihood ratio test and rejected in almost all cases (see Section 3). Therefore, divergence times were estimated using multidivtime, which implements a 'relaxed clock' model of rate variation. This model is best described as a Brownian motion process that operates on the logarithm of the rates of molecular evolution (Thorne et al., 1998). Rates are assumed to be constant on a particular branch of a tree but they are allowed to vary among different branches. The autocorrelation between the rates on an ancestral branch and its descendent depends on the time elapsed between those branches. To enable inference under this parameter-rich model Thorne et al. (1998) have adopted a Bayesian framework. MULTIDIVTIME allows information from multiple genes to be combined and also tests for correlated changes in evolutionary rates among genes (Kishino et al., 2001; Thorne and Kishino, 2002; Thorne et al., 1998). MULTIDIVTIME uses a Metropolis-Hastings Markov Chain Monte Carlo (MCMC) algorithm to obtain posterior estimates of evolutionary rates and of the dates of each node in a phylogeny. MCMC methods allow simulating a Markov chain process with a stationary distribution that is the probability density of interest. More specifically, the Metropolis-Hastings algorithm simulates sequential values from this chain until it converges on the stationary distribution (the 'burn-in' period). The algorithm then samples values from the state of the chain at regular intervals to approximate independent samples from the stationary 
distribution (Hastings, 1970; Metropolis et al., 1953). We calculated a marginal probability distribution for the dates of the calibration nodes (see Table 2 for more algorithm details) from 10,000 states sampled every 500th state after burn-in.

The relaxed clock model was calibrated by constraining the divergence time for a node in the PTLV tree to a specified time interval that represents the upper and lower limits of a hypothesized anthropological migration event. The most commonly used calibration of the PTLV phylogeny is based on the early migration of Melanesian settlers (the 'Melanesian' calibration). According to genetic and archeological evidence, this migration from Asia to Melanesia occurred 40,000-60,000 years ago. This time-interval includes uncertainty in the time of the earliest migration to these islands (Cavalli-Sforza et al., 1994; Roberts et al., 1990; Van Dooren et al., 2001). However, there is another putative calibration node in the PTLV phylogeny: HTLV-2 is assumed to have an ancient African origin and several investigators have suggested that the virus among native American Indians was brought originally from Asia into the Americas 15,000-35,000 years ago during the migration of infected Asian populations over the Bering land bridge (Biggar et al., 1996; Neel et al., 1994; Suzuki and Gojobori, 1998). This event may correspond to the common ancestor of the HTLV-2a and HTLV-2b subtypes (the 'Bering' calibration).

\subsection{Statistical model of HTLV evolutionary rate}

We developed a Bayesian statistical model, with evolutionary rate as its central variable, to combine and compare the information obtained from the anthropological and pedigree data sets. The essence of a Bayesian approach is to calculate how the observation of new evidence (data) changes our existing belief (prior) in a certain hypothesis. Here, we will consider the results of the anthropological calibration analysis as our prior belief, and the pedigree data as our new evidence.

Evolutionary rates vary across the PTLV genome. Therefore exactly the same genome region must be used in the anthropological and pedigree analyses in order to combine and compare the results in a valid manner. To this end, we repeated the anthropological analysis (as described above) using the exact same gene regions as used in the pedigree study (Van Dooren et al., 2004). This resulted in LTR and env datasets that contained 50 and 68 strains, respectively. As before, the molecular clock hypothesis was tested and rejected, so evolutionary rate estimates were obtained using MULTIDIVTIME. The relaxed molecular clock was calibrated by constraining the divergence time for the HTLV-1c subtype (MEL5) to 40,000-60,000 years ago. MULTIDIVTIME was used to calculate a marginal posterior probability distribution for the average evolutionary rate (obtained from 2000 states of posterior rates at each node, sampled every 5000th state after burn-in).
A gamma distribution was then fitted to the MULTIDIVTIME marginal posterior density (using maximum likelihood). This gamma distribution represents our prior information about HTLV evolutionary rate in the Bayesian statistical model. If $\lambda$ denotes evolutionary rate then:

$\lambda \sim \operatorname{Gamma}(\alpha, \beta)$

where $\alpha$ and $\beta$ are the parameters of the gamma distribution, and " $\sim$ " represents "is distributed according to".

To incorporate Van Dooren et al.'s (2004) data we used a homogenous Poisson process to model the number substitutions $(Y)$ that were observed in the pedigree sequence data (see Van Dooren et al., 2004 for more details). Therefore:

$Y \sim \operatorname{Poisson}(\lambda t) I\left(Y_{\text {low }}, Y_{\text {up }}\right)$

where $t$ denotes the number of years represented in the pedigrees, and $I$ represents an indicator function that censors the Poisson distribution. The indicator function is needed because some of the mutations in the pedigree analysis were ambiguous. The lower limit $\left(Y_{\text {low }}\right)$ excludes the ambiguous mutations whilst the upper limit $\left(Y_{\text {up }}\right)$ includes them as additional substitutions. In addition, the number of years represented by the pedigrees was only known to lie within a certain range, from $t_{\text {low }}=419$ to $t_{\text {up }}=1101$ years. Therefore, the number of years $t$ is modeled as a covariate, with an interval-censored normal distribution:

$t \sim \operatorname{Normal}(\mu, \sigma) I\left(t_{\text {low }}, t_{\text {up }}\right)$

In our analysis, mean $\mu=680$ years and S.D. $\sigma=100,000$.

To obtain a posterior distribution for the evolutionary rate that combines both the pedigree and the anthropological data, we used MCMC, as implemented in WINBUGS v1.4 (http://www.mrc-bsu.cam.ac.uk/bugs/). Convergence was investigated in CODA (http://www.mrc-bsu.cam.ac.uk/ bugs/classic/coda04/readme.shtml).

\section{Results}

\subsection{Consistency of anthropological dates}

We used full genome PTLV sequences to investigate whether the assumed dates for the 'Melanesian' and 'Bering' migration events were consistent with each other when applied to the PTLV phylogeny. As shown in Table 1, the molecular clock was tested for each gene and for the full genome (Table 1). The molecular clock hypothesis was consistently rejected (except for the tax gene). Interestingly, there is a significant correlation between alignment length and the likelihood that the clock is rejected, for both nucleotide $\left(P<0.01, R^{2}=0.93\right)$ and amino acid $(P<0.01$, $\left.R^{2}=0.98\right)$ alignments. In the absence of clocklike evolution, we used a Bayesian approach that implements a relaxed clock model and is capable of handling multigene data sets (Thorne and Kishino, 2002). 
Table 1

Molecular clock tests

\begin{tabular}{lcccc}
\hline Genome region & Nucleotides & LRS & Amino acids & LRS \\
\hline gag & 1296 & $59.2^{\mathrm{a}}$ & 432 & $48.6^{\mathrm{a}}$ \\
prot & 537 & $65.45^{\mathrm{a}}$ & 179 & $45.1^{\mathrm{a}}$ \\
pol & 2601 & $104.2^{\mathrm{a}}$ & 867 & $76.0^{\mathrm{a}}$ \\
env & 1461 & $78.2^{\mathrm{a}}$ & 487 & $62.8^{\mathrm{a}}$ \\
tax & 981 & 27.9 & 327 & $45.8^{\mathrm{a}}$ \\
Full genome & 6817 & $211.2^{\mathrm{a}}$ & 2246 & $132.4^{\mathrm{a}}$ \\
\hline
\end{tabular}

The likelihood ratio statistic (LRS) is defined as twice the difference in log likelihood under the clock and different-rates models. Number in the nucleotide and amino acid columns indicates the length of the input alignment.

${ }^{a}$ Indicates a significant rejection of the molecular clock hypothesis $(P<0.05)$.

Fig. 1 shows the PTLV phylogeny used to estimate divergence times. When the molecular clock was calibrated using the dates for the 'Melanesian' migration event clade, the estimated date for the 'Bering' calibration node was $26,860(19,000-36,930)$ years ago (Table 2$)$. When the dates for the 'Bering' migration were constrained between 15,000-35,000, the estimated date for the 'Melanesian' calibration node was 44,980 $(26,340-72,420)$ years ago. Hence, the calibrations appear to be compatible. Not surprisingly, the confidence intervals for the estimated dates are reduced when both constraints were applied simultaneously (Table 2). It is interesting to note that in the multigene data set, no pair of genes had significantly correlated evolutionary changes.

\subsection{Statistical model of HTLV evolutionary rate}

We performed a Bayesian analysis to combine and compare pedigree-based and anthropology-based estimates of HTLV evolutionary rate. To obtain a comparable anthropology-based estimate, we repeated the relaxed-clock MULTIDIVTIME analysis using the exact same HTLV-1 genome regions as in the pedigree study (Van Dooren et al., 2004). The single-rate molecular clock was significantly rejected for both LTR and env data sets. The relaxed clock was calibrated by constraining the date of the 'Melanesian' calibration node to 40,000-60,000 years ago. This resulted in an estimated rate of $5.6 \times 10^{-7}\left(1.2 \times 10^{-7}\right.$ to $\left.1.1 \times 10^{-6}\right)$ and $2.1 \times 10^{-7}\left(2.1 \times 10^{-8}\right.$ to $\left.4.5 \times 10^{-7}\right)$ substitutions/site/year for LTR and env, respectively. These estimates are in good agreement with previous calculations based on the same calibration (Salemi et al., 2000; Yanagihara et al., 1995). Fig. 2A shows the marginal posterior probability histogram for LTR evolutionary rate that was calculated by MULTIDIVTIME. As can be seen, the fitted gamma function provides a very good approximation to this distribution. A similar result was obtained for the env region (data not shown).

A Bayesian statistical model was used to combine the MULTIDIVTIME result with the results of the pedigree study, whilst taking into account the various sources of statistical uncertainty in the data. Fig. 2B and $\mathrm{C}$ show plots of the prior density, the likelihood and the posterior density of the LTR and $e n v$ evolutionary rates, respectively, under our statistical model calculated using MCMC in WinBugs. The prior and the likelihood are consistent with each other, in that the latter considerably overlaps the former. However, the posterior density varies little from the anthropologybased prior. The small sample size of the pedigree-based analysis has resulted in an evolutionary rate estimate with a very large variance in comparison to the anthropology-based analysis.

This result leads us to investigate what sample size would be necessary in a pedigree study in order to produce an estimate with approximately the same statistical power as the current anthropology-based estimate. To do this, we used WinBugs to simulate adding extra mutations to the pedigree study env dataset (whilst constraining the pedigree rate estimate to its original value by appropriately increasing $t$ ). For each additional simulated mutation, we explored how the posterior mean changed. As Fig. 1D shows, approximately 75 extra mutations would be required in the pedigree study in order to shift the posterior mean to halfway between the current anthropology-based and pedigree-based estimates. To obtain this number of mutations, the pedigree study would need to include about 300 vertical transmission chains.

\section{Discussion}

The analyses presented in this study were aimed at achieving two highly related goals: (i) investigating the compatibility of alternative anthropological calibrations in the PTLV phylogeny; and (ii) investigating the compatibility of phylogeny-based estimates of the HTLV evolutionary rate, using anthropological calibrations, and pedigree-based estimates. Under the hypothesis of codivergence, different migration-based calibrations should be consistent with each other. Several epidemiological investigations have suggested that HTLV-2 (subtypes $a$ and $b$ ) isolated from Amerindian tribes was originally brought into the Americas during the Bering land bridge migrations 15,000-35,000 years ago (Biggar et al., 1996; Neel et al., 1994; Suzuki and Gojobori, 1998). Our analysis shows that this timeframe is roughly equal to the confidence interval of the phylogenetic divergence time for HTLV-2a and HTLV-2b. Constraining this node to $15,000-35,000$ years ago resulted in an estimated date for the early migration of Melanesian settlers of 44,980 $(26,340-72,420)$ years ago, again consistent with anthropological information. Therefore, we propose a timescale based on both calibrations (Fig. 1). The consistency of these two calibrations has been suggested before, using less accurate methods (Salemi et al., 2000); our estimate for the PTLV time of origin is more recent than that previous estimate. It has been frequently shown that HTLV does not evolve according to a molecular clock, forcing 

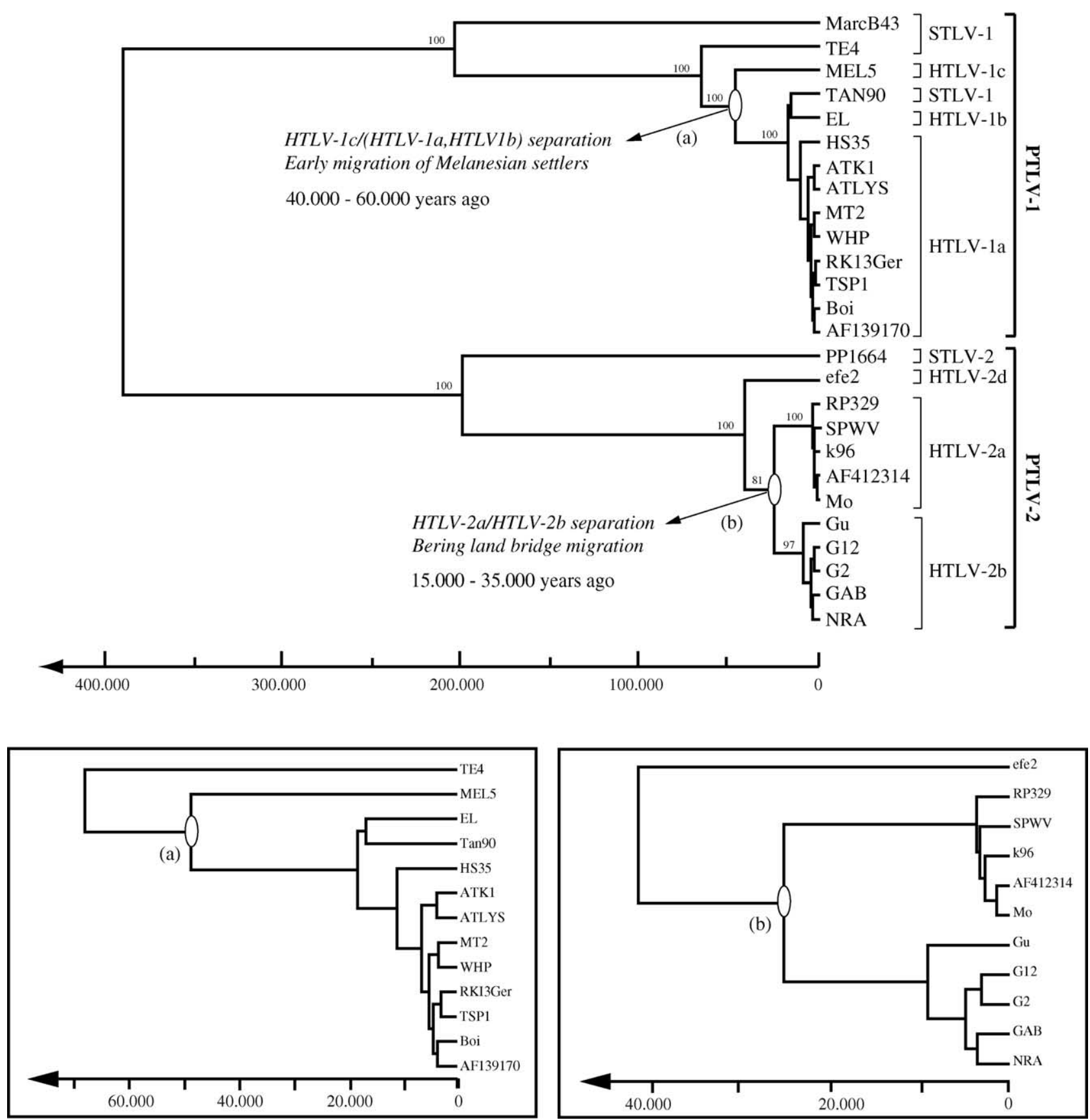

Fig. 1. Maximum likelihood tree for 26 full-genome PTLV strains. Types and subtypes of the viral strains are indicated at the tips of the tree. Numbers at the nodes indicate the percentage of bootstrap samples (1000 replicates) in which the node is supported (only values $>80 \%$ are shown). The 'Melanesian' and 'Bering' calibration nodes used in the relaxed molecular clock analysis are indicated with arrows. The time-scale for the tree was calculated by MULTIDIVTIME using both calibration points. More detailed time-scales for the PTLV-1 and PTLV-2 clades separately are shown below the global time-scale.

Table 2

Dates estimated using the anthropological calibrations

\begin{tabular}{lll}
\hline Anthropological calibration(s) used & Estimated date of 'Melanesian' node (a) & Estimated date of 'Bering' node (b) \\
\hline Melanesian migration event & $49400(40400-59400)$ & $26860(19000-36930)$ \\
Bering migration event & $44980(26340-72420)$ & $23810(15280-34350)$ \\
Melanesian and Bering migration events & $49130(40350-59220)$ & $26220(19030-33900)$ \\
\hline
\end{tabular}

(a) and (b) refer to the nodes in Fig. 1. The chains in MULTIDIVTIME were run for $5 \times 10^{6}$ states and were sampled every 500th state after burn-in (up to $10^{5}$ states). The prior for the substitution rate was normal (mean $=10^{-7}$ substitutions/site/year; S.D. $=0.0001$ ). The prior for the root height was also normal $\left(\right.$ mean $=10^{6}$ years ago; S.D. $\left.=10^{9}\right)$. 


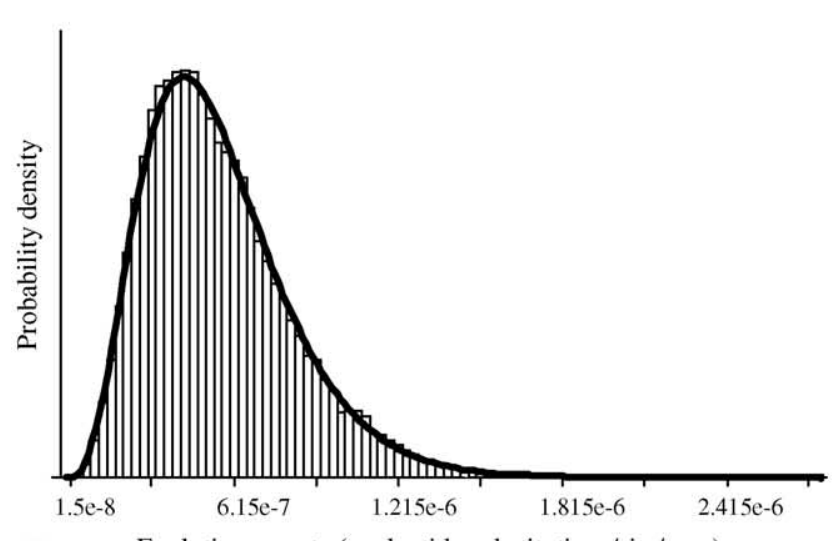

(A) Evolutionary rate (nucleotide substitutions/site/year)

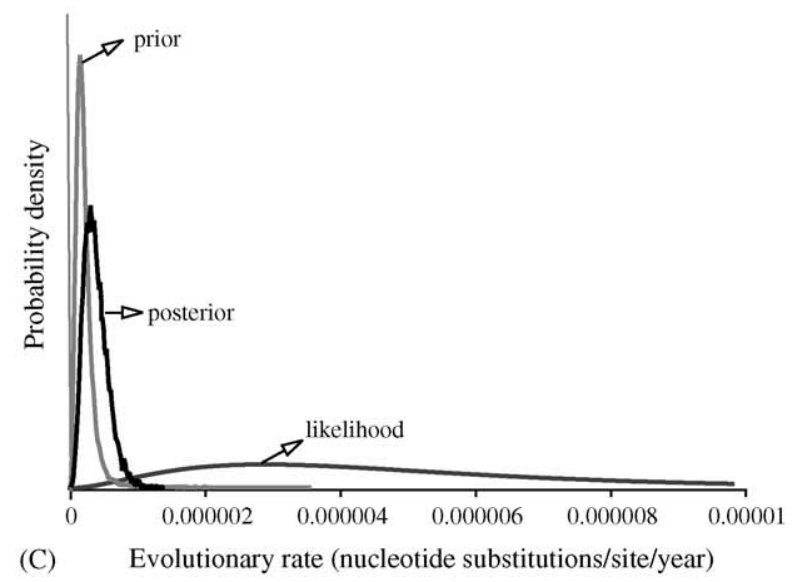

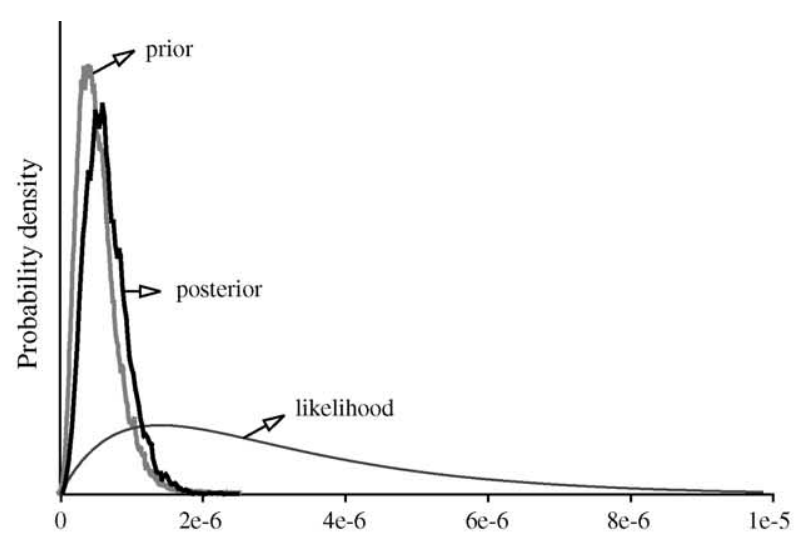

(B) Evolutionary rate (nucleotide substitutions/site/year)

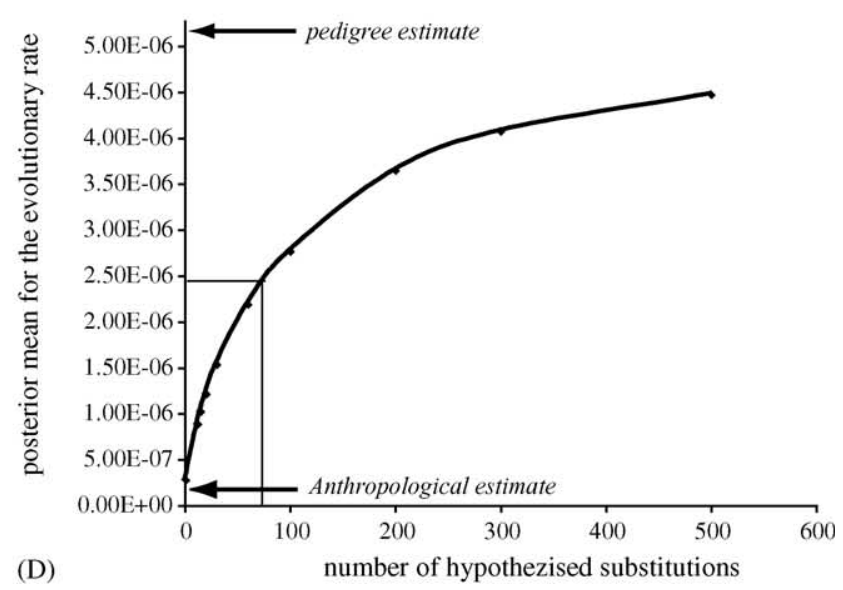

Fig. 2. Results of the Bayesian evaluation of HTLV evolutionary rate. (A) Histogram representing the marginal posterior probability for the HTLV LTR evolutionary rate, obtained using MULTIDIVTIME. The black curve shows the gamma distribution fitted using maximum likelihood. (B) and (C) Results of the Bayesian statistical model for HTLV evolutionary rate in LTR and env, respectively. The three curves represent: (i) the anthropology-based prior density (see A (ii) the likelihood curve calculated from the pedigree data, and (iii) the resulting posterior density. (D) Plot showing how the mean posterior evolutionary rate increases as the number of simulated pedigree mutations increases. The number of years represented by the pedigrees was allowed to increase so that the evolutionary rate estimate for the pedigree data remained constant, at $5.15 \times 10^{-6}$ substitutions/site/year.

previous analyses to discard information inconsistent with this null hypothesis (Meertens and Gessain, 2003; Meertens et al., 2003; Salemi et al., 2000; Van Dooren et al., 2001). In our study, the molecular clock was significantly rejected for all genes except for tax, and the longer the input alignment, the more likely the rejection of the clock hypothesis. This suggests that if a single-rate clock is used then more data must be ignored in larger data sets; e.g. limiting the analysis to the third codon positions only (Meertens and Gessain, 2003; Salemi et al., 2000). To resolve these problems we used a Bayesian method that relaxes the rate-constancy assumption; this method might be more appropriate for the large multigene PTLV data set. An additional advantage of the Bayesian framework is the ability to constrain nodes to a range of times, thereby taking into account uncertainty in the date of the anthropological migration event.

Ideally, we would like to use calibrations of viral phylogenies that are independent of anthropological hypotheses, but these are difficult to obtain for slowly evolving viruses. A recent study of vertical HTLV-1 transmission of in several families tried to address this problem (Van Dooren et al., 2004). Here, we have developed a Bayesian statistical model to combine and compare the pedigree data and the anthropology-based estimates. We investigated whether observation of the pedigree data requires us to update our belief in the anthropology-based prior and showed that the anthropology-based prior has much less variance than the pedigree data and therefore, strongly dominates the posterior density. Therefore, we can conclude that the pedigree estimate does not have enough statistical power to provide an alternative to the anthropological calibrations The use of the anthropology-based estimate as a prior is arbitrary-our conclusions remain the same if the pedigree estimate is the prior and the anthropology-based estimate is the data. Using simulation we showed, for the env data set, that the number of HTLV families investigated should increase by 40 -fold if the pedigree analysis is to be as informative as a molecular clock analysis. It should also be noted that pedigree data results from vertical transmission only. It has been shown that 
horizontal HTLV transmission, most notably in injecting drug user populations, can lead to increased substitution rates (Salemi et al., 1999). Therefore, a phylogeny-based evolutionary rate estimate will be a composite one (Holmes, 2003). Our estimates for the evolutionary rate resulted in a posterior mean of $3.44 \times 10^{-7}$ (confidence interval of $1.091 \times 10^{-7}$ to $7.118 \times 10^{-7}$ ) substitutions/site/year and $6.55 \times 10^{-7}$ (confidence interval of $2.248 \times 10^{-7}$ to $1.31 \times 10^{-6}$ ) substitutions/site/year for env and LTR, respectively. Not surprisingly, these rates are very similar to previous phylogeny-based estimates (Song et al., 1994; Yanagihara et al., 1995). Other retroviruses, like HIV, Simian Immunodeficiency Virus, Murine Leukemia Virus, Equine Infectious Anemia Virus and Rous Sarcoma Viruses have rates in the order of $10^{-3}$ substitutions/site/year (Burns and Desrosiers, 1991; Clements et al., 1988; Suzuki et al., 2000). This difference has been explained by the replication of HTLV proviral genomes by host DNA polymerases, resulting in high-fidelity copies of the HTLV genome (Wattel et al., 1995).

The question as to what extent HTLV evolution has been shaped by human migrations is far from resolved. A more extensive sampling of HTLV genetic diversity worldwide is essential to address this problem. Although PTLV phylogenies calibrated with human migration dates result in relatively precise estimates for the evolutionary rate, it remains difficult to evaluate their accuracy since they rely on an anthropological assumption that may not be correct. Further research is necessary to estimate the probability of the anthropological hypotheses used; this uncertainty should then be added to the Bayesian statistical model developed here. Pedigree-based data is important, as it is anthropologyindependent. However, much more data is needed to obtain reliable evolutionary rate estimates. So, there is a problem of uncertainty for both types of information: the large variance of current pedigree data and the underlying assumption of anthropological calibrations. Our analysis indicates that the pedigree data does not urge for a different point of view on the HTLV evolutionary rate. However, it should be noted that the pedigree data at hand would not urge for a different point of view on many different HTLV prior specifications. For example, the estimated rate from the pedigree data would most probably not discriminate between pre- or postColumbian introduction of HTLV-1 subtype in Latin America (Miura et al., 1994; Van Dooren et al., 1998). However, the probabilistic framework developed here might be useful in addressing similar scenarios for other slowly evolving viruses.

\section{Acknowledgements}

We thank A. Komarek for assistance with the Winbugs analyses. This work was supported by the Flemish Fonds voor Wetenschappelijk Onderzoek (FWO G.0288.01); P.L. was supported by the Institute for the Promotion of
Innovation through Science and Technology in Flanders (IWT Vlaanderen); O.G.P. was supported by the Welcome Trust.

\section{References}

Biggar, R.J., Taylor, M.E., Neel, J.V., Hjelle, B., Levine, P.H., Black, F.L., Shaw, G.M., Sharp, P.M., Hahn, B.H., 1996. Genetic variants of human T-lymphotrophic virus type II in American Indian groups. Virology 216, $165-173$.

Burns, D.P., Desrosiers, R.C., 1991. Selection of genetic variants of simian immunodeficiency virus in persistently infected rhesus monkeys. J. Virol. 65, 1843-1854.

Cavalli-Sforza, L., Menozzi, L., Piazza, A., 1994. The History and Geography of Human Genes. Princeton University Press, Princeton, N.J..

Clements, J.E., Gdovin, S.L., Montelaro, R.C., Narayan, O., 1988. Antigenic variation in lentiviral diseases. Annu. Rev. Immunol. 6, 139-159.

Drummond, A.J., Nicholls, G.K., Rodrigo, A.G., Solomon, W., 2002. Estimating mutation parameters, population history and genealogy simultaneously from temporally spaced sequence data. Genetics 161, 1307-1320.

Drummond, A.J., Pybus, O.G., Rambaut, A., Forsberg, R., Rodrigo, A.G., 2003. Measurably evolving populations. Trends Ecol. Evol. 18, 481488.

Gessain, A., Gallo, R.C., Franchini, G., 1992. Low degree of human T-cell leukemia/lymphoma virus type I genetic drift in vivo as a means of monitoring viral transmission and movement of ancient human populations. J. Virol. 66, 2288-2295.

Hastings, W.K., 1970. Monte Carlo sampling methods using Markov chains and their applications. Biometrika 57, 97-109.

Holmes, E.C., 2003. Molecular clocks and the puzzle of RNA virus origins. J. Virol. 77, 3893-3897.

Holmes, E.C., Zhang, L.Q., Simmonds, P., Ludlam, C.A., Brown, A.J., 1992. Convergent and divergent sequence evolution in the surface envelope glycoprotein of human immunodeficiency virus type 1 within a single infected patient. Proc. Natl. Acad. Sci. U.S.A. 89, 4835-4839.

Huelsenbeck, J.P., Larget, B., Swofford, D., 2000. A compound Poisson process for relaxing the molecular clock. Genetics 154, 1879-1892.

Kishino, H., Thorne, J.L., Bruno, W.J., 2001. Performance of a divergence time estimation method under a probabilistic model of rate evolution. Mol. Biol. E 18, 352-361.

Korber, B., Muldoon, M., Theiler, J., Gao, F., Gupta, R., Lapedes, A., Hahn, B.H., Wolinsky, S., Bhattacharya, T., 2000. Timing the ancestor of the HIV-1 pandemic strains. Science 288, 1789-1796.

Lemey, P., Pybus, O.G., Wang, B., Saksena, N.K., Salemi, M., Vandamme, A.M., 2003. Tracing the origin and history of the HIV-2 epidemic. Proc. Natl. Acad. Sci. U.S.A. 100, 6588-6592.

Liu, H.F., Goubau, P., Van, B., Van, L., Chen, Y.C., Desmyter, J., Vandamme, A.M., 1996. The three human T-lymphotropic virus type I subtypes arose from three geographically distinct simian reservoirs. J. Gen. Virol. 77 (Pt 2), 359-368.

McGeoch, D.J., Dolan, A., Ralph, A.C., 2000. Toward a comprehensive phylogeny for mammalian and avian herpesviruses. J. Virol. 74, 1040110406.

Meertens, L., Gessain, A., 2003. Divergent simian T-cell lymphotropic virus type 3 (STLV-3) in wild-caught Papio hamadryas papio from Senegal: widespread distribution of STLV-3 in Africa. J. Virol. 77, 782-789.

Meertens, L., Shanmugam, V., Gessain, A., Beer, B.E., Tooze, Z., Heneine, W., Switzer, W.M., 2003. A novel, divergent simian T-cell lymphotropic virus type 3 in a wild-caught red-capped mangabey (Cercocebus torquatus torquatus) from Nigeria. J. Gen. Virol. 84, 2723-2727.

Metropolis, N., Rosenbluth, A.W., Rosenbluth, M.N., Teller, A.H., Teller, E., 1953. Equation of state calculations by fast computing machines. J. Chem. Phys. 21, 1087-1092. 
Miura, T., Fukunaga, T., Igarashi, T., Yamashita, M., Ido, E., Funahashi, S., Ishida, T., Washio, K., Ueda, S., Hashimoto, K., 1994. Phylogenetic subtypes of human T-lymphotropic virus type I and their relations to the anthropological background. Proc. Natl. Acad. Sci. U.S.A. 91, 11241127.

Neel, J.V., Biggar, R.J., Sukernik, R.I., 1994. Virologic and genetic studies relate Amerind origins to the indigenous people of the Mongolia/ Manchuria/southeastern Siberia region. Proc. Natl. Acad. Sci. U.S.A. 91, 10737-10741.

Pybus, O.G., Charleston, M.A., Gupta, S., Rambaut, A., Holmes, E.C., Harvey, P.H., 2001. The epidemic behavior of the hepatitis $\mathrm{C}$ virus. Science 292, 2323-2325.

Pybus, O.G., Drummond, A.J., Nakano, T., Robertson, B.H., Rambaut, A., 2003. The epidemiology and iatrogenic transmission of hepatitis $C$ virus in Egypt: a Bayesian coalescent approach. Mol. Biol. E 20, 381-387.

Pybus, O.G., Rambaut, A., 2002. GENIE: estimating demographic history from molecular phylogenies. Bioinformatics 18, 1404-1405.

Rambaut, A., 2000. Estimating the rate of molecular evolution: incorporating non-contemporaneous sequences into maximum likelihood phylogenies. Bioinformatics 16, 395-399.

Roberts, R.G., Jones, R., Smith, M.A., 1990. Report of thermoluminescence dates supporting the arrival of people between 50 and 60 kya in southern Australia. Nature 345, 153.

Salemi, M., Desmyter, J., Vandamme, A.M., 2000. Tempo and mode of human and simian T-lymphotropic virus (HTLV/STLV) evolution revealed by analyses of full-genome sequences. Mol. Biol. E 17, 374-386.

Salemi, M., Lewis, M., Egan, J.F., Hall, W.W., Desmyter, J., Vandamme, A.M., 1999. Different population dynamics of human T cell lymphotropic virus type II in intravenous drug users compared with endemically infected tribes. Proc. Natl. Acad. Sci. U.S.A. 96, 13253-13258.

Salemi, M., Van, D., Audenaert, E., Delaporte, E., Goubau, P., Desmyter, J., Vandamme, A.M., 1998. Two new human T-lymphotropic virus type I phylogenetic subtypes in seroindeterminates, a Mbuti pygmy and a Gabonese, have closest relatives among African STLV-I strains. Virology 246, 277-287.

Shankarappa, R., Gupta, P., Learn, G.H., Rodrigo, A.G., Rinaldo, C.R., Gorry, M.C., Mullins, J.I., Nara, P.L., Ehrlich, G.D., 1998. Evolution of human immunodeficiency virus type 1 envelope sequences in infected individuals with differing disease progression profiles. Virology 241, 251-259.

Song, K.J., Nerurkar, V.R., Saitou, N., Lazo, A., Blakeslee, J.R., Miyoshi, I., Yanagihara, R., 1994. Genetic analysis and molecular phylogeny of simian T-cell lymphotropic virus type I: evidence for independent virus evolution in Asia and Africa. Virology 199, 56-66.

Strimmer, K., von Haeseler, A., 1996. Quartet puzzling: a quartet maximum likelihood method for reconstructing tree topologies. Mol. Biol. E 13, 964-969.

Suzuki, Y., Gojobori, T., 1998. The origin and evolution of human T-cell lymphotropic virus types I and II. Virus Genes 16, 6984.

Suzuki, Y., Yamaguchi-Kabata, Y., Gojobori, T., 2000. Nucleotide substitution rates of HIV-1. AIDS Rev. 2, 39-47.

Swofford, D.L., 1998. PAUP 4.0-Phylogenetic Analysis Using Parsimony (and other methods). Sinauer Assoc., Sunderland, MA.

Thorne, J.L., Kishino, H., 2002. Divergence time and evolutionary rate estimation with multilocus data. Syst. Biol. 51, 689-702.

Thorne, J.L., Kishino, H., Painter, I.S., 1998. Estimating the rate of evolution of the rate of molecular evolution. Mol. Biol. E 15, 16471657.

Van Dooren, S., Gotuzzo, E., Salemi, M., Watts, D., Audenaert, E., Duwe, S., Ellerbrok, H., Grassmann, R., Hagelberg, E., Desmyter, J., Vandamme, A.M., 1998. Evidence for a post-Columbian introduction of human T-cell lymphotropic virus [type I] [corrected] in Latin America. J. Gen. Virol. 79 (Pt 11), 2695-2708.

Van Dooren, S., Pybus, O.G., Salemi, M., Liu, H.F., Goubau, P., Remondegui, C., Talarmin, A., Gotuzzo, E., Alcantara, L.C., Galvao-Castro, B., Vandamme, A.M., 2004. The low evolutionary rate of human T-cell lymphotropic virus type- 1 confirmed by analysis of vertical transmission chains. Mol. Biol. E 21, 603-611.

Van Dooren, S., Salemi, M., Vandamme, A.M., 2001. Dating the origin of the African human T-cell lymphotropic virus type-i (HTLV-I) subtypes. Mol. Biol. E 18, 661-671.

Vandamme, A.M., Liu, H.F., Goubau, P., Desmyter, J., 1994. Primate Tlymphotropic virus type I LTR sequence variation and its phylogenetic analysis: compatibility with an African origin of PTLV-I. Virology 202, 212-223.

Wattel, E., Vartanian, J.P., Pannetier, C., Wain, H., 1995. Clonal expansion of human T-cell leukemia virus type I-infected cells in asymptomatic and symptomatic carriers without malignancy. J. Virol. 69, 2863 2868.

Yanagihara, R., Saitou, N., Nerurkar, V.R., Song, K.J., Bastian, I., Franchini, G., Gajdusek, D.C., 1995. Molecular phylogeny and dissemination of human T-cell lymphotropic virus type I viewed within the context of primate evolution and human migration. Cell Mol. Biol. 41 (Suppl. 1), S145-S161. 